\title{
Aucune part, évolution diachronique et nature de son changement linguistique
}

\author{
Dhoukar, Asma \\ MoDyCo, Paris Ouest Nanterre La Défense \\ asma141982@yahoo.fr
}

\section{Introduction}

Part, d'après l'étude des articles que lui consacrent plusieurs dictionnaires ${ }^{1}$ suivant une perspective diachronique, à savoir le Godefroy dictionnaire (désormais GD), le Godefroy complément $(G C)$, le dictionnaire du moyen français $(D M F)$, le dictionnaire Huguet $(D H)$, le dictionnaire Littré $(D L)$ et le Trésor de la langue française informatisé (TLFi), se caractérise par une diversité sémantique et lexicale importante. Il présente des emplois grammaticalisés et entre également et surtout dans toutes sortes de constructions lexicales, qu'elles soient figées ou non, faisant de lui un « mot-caméléon ». Nous l'appelons ainsi parce qu'il s'intègre facilement dans des domaines variés (arithmétique, astrologie, commerce, diplomatie, droit, grammaire, politique, domaine marin, théâtre...), se caractérise par une richesse et une variation sémantique importante (nous avons relevé principalement les notions suivantes : « communication et renseignement», « origine, extraction, qualité », « affection », " parti, faction», «côté, direction», «lieu, endroit, région», «partie d'un tout avec/sans idée de partage », « fraction/portion d'un patrimoine (notion d'attribution)» et «participation ») et figure dans différentes locutions (adjectivales, adverbiales, conjonctives, prépositionnelles et verbales (avoir part, donner part, faire part et prendre part)) contribuant à son figement dans de nombreuses constructions.

Cette double fonctionnalité de part a été mentionnée par exemple par Blanche-Benveniste (2003 : 286), qui distingue l'emploi grammaticalisé de part (quelque part) de son emploi lexical (de part et d'autre, être à part), deux emplois qu'elle caractérise comme tout à fait différents.

Suivant l'étude de Blanche-Benveniste (Ibid.) sur la grammaticalisation de quelque part, nous poserons ici la question de la nature du changement linguistique qu'a subi aucune part: s'agit-il d'un cas de grammaticalisation, de lexicalisation ou d'un autre type d'évolution ? Est-ce qu'il serait abusif de parler de grammaticalisation dans la mesure où elle exige, par exemple, un changement de catégorie grammaticale (passage du lexical vers le grammatical) et plus prudent d'opter pour la lexicalisation « qui met davantage l'accent sur la forme cible, lexicale, sans présumer de la forme d'origine, lexicale ou grammaticale, ni même du processus (si la forme de départ est lexicale, la forme d'arrivée n'est pas nécessairement «plus» lexicale)» (Prévost, 2006: 128). Pour répondre à cette question, nous considérons d'abord les caractéristiques morphosyntaxiques de aucune part qui, sur certains points, montrent que dans des états antérieurs de la langue la locution ne connaît pas le même degré de figement que dans le français actuel, du moins dans son emploi courant : l'emploi du pluriel (aucunes pars), l'insertion d'un adjectif/indéfini entre aucune et part (aucune vraie part, aucune autre part), l'accord de l'adjectif joint à part (aucune part bonne ou mauvaise) et la reprise en anaphore (aucune part que celle). Au plan sémantique, aucune part se caractérise, suite à son emploi dans différentes constructions (dont principalement avoir aucune part et prendre aucune part), par une variation sémantique qui se résume généralement dans les notions suivantes: «lieu», «partie d'un tout» et «participation». Des observations morphosyntaxiques et sémantiques que nous utiliserons finalement pour déterminer la nature du changement subi par aucune part.

Pour mener à bien cette étude, nous avons utilisé un corpus suivant une perspective diachronique, formé à partir de la Base du Français Médiéval ${ }^{2}$ (du IX ${ }^{\mathrm{e}}$ jusqu'au début du XIV siècle), de la base du Dictionnaire du Moyen Français ${ }^{3}\left(\mathrm{du} \mathrm{XIV}^{\mathrm{e}}\right.$ jusqu'au début du $\mathrm{XVI}^{\mathrm{e}}$ siècle) et de Frantext ${ }^{4}$ pour les siècles suivants (du XVI ${ }^{\mathrm{e}}$ jusqu'au $\mathrm{XX}^{\mathrm{e}}$ siècle). 


\section{Aucune part, caractéristiques morphosyntaxiques}

Le syntagme aucune part, dont notre corpus nous a révélé 518 attestations réparties entre son emploi avec le sens spatial de «quelque part»/«nulle part» et son emploi dans les locutions verbales avec verbe support (faire, prendre, avoir part...), est doté de plusieurs caractéristiques morphosyntaxiques, qui manifestent une divergence de fonctionnement entre états antérieurs du français : l'emploi au pluriel (aucunes pars), la possibilité d'insertion d'un adjectif/indéfini entre aucune et part (aucune vraielautre part), la reprise en anaphore (aucune part...celle) et l'accord au féminin suite à la construction directe avec un adjectif (aucune part mauvaise).

\section{$2.1 \quad$ L'emploi du pluriel}

Aucune part se caractérise par sa variation en nombre, si nous tenons compte de la seule attestation révélée par notre corpus où aucunes pars évoque un « lieu figuré »:

(1) Et mesmement nous vivans sommes, En aucunes pars, soubz les sommes Et la discipline des nombres, (De Pizan, Le Livre de la Mutacion de Fortune T.2, 1400 : 115)

\subsection{Les cas d'insertion}

Notre construction se caractérise également par la possibilité d'insertion d'un indéfini/adjectif entre aucune et part. Nous avons repéré deux types de structure à savoir aucune + autre part et aucune + vraie part. Dans le premier cas, aucune se joint à l'expression autre part exprimant le «lieu » («nulle part») dans une représentation abstraite d'un lignage :

(2) sur la teste des enfans de feu Madame Claude de France, sa fille, ne voiant apparence de lignée de sa race, d'aucune autre part. (L'estoile, Registre-journal du regne de Henri III : t. 5 (1585-1587), $1587: 35-36)$

Dans l'exemple (3), l'adjectif vraie inséré entre aucune et part se trouve dans la construction avoir part : n'avoir aucune vraie part aux essais au sens de « ne pas contribuer, ne pas participer »:

(3) Le quatriesme est qu'ils n'ont aucune vraye part aux essais que les hommes font les uns contre les autres par jalousie d'honneur et de valeur, aux exercices de l'esprit ou du corps, qui est une des plus plaisantes choses qui soit au commerce des hommes. (Charron, De la sagesse : trois livres, $1601: 215-216$ )

\subsection{La reprise en anaphore}

Une autre caractéristique concerne la possibilité de sa reprise en anaphore par celle au XVII siècle. Aucune part, lexicalisé dans la langue classique, évoque ici la notion de «partie d'un tout abstrait: part du gouvernement » :

(4) il assembla la noblesse, lui mit le gouvernement entre les mains, et protestant qu'il n'y prétendoit aucune part que celle qui lui seroit commune avec tous les autres gentilshommes, il donna lui-même la forme à la république, (Retz, La Conjuration $d u$ comte Jean-Louis de Fiesque, 1665 : 509-510)

Une reprise qui reste possible au $\mathrm{XIX}^{\mathrm{e}}$ siècle grâce à celle et part :

(5) J'ai vu de tous les côtés les intérêts et les passions qui les ont continués, et par la grâce de Dieu je n'y ai pris aucune part que celle de m'en affliger, d'en gémir devant Dieu et de le prier d'inspirer des sentiments de paix et de charité à ceux qui paraissent en avoir de tout contraires. (Chateaubriand, Vie de Rancé, 1844 : 189-190)

(6) Tout cela sans préjudice de tout le reste du devoir. Je ne veux aucune part du pouvoir, mais je veux part entière au danger. (Hugo, Correspondance : $t .3: 1867-1873,1873$ : 265-266) 


\subsection{Construction directe et accord de l'adjectif joint à aucune part}

Une dernière caractéristique concerne l'accord au féminin suite à la construction directe de l'adjectif joint à aucune part. En témoignent des attestations repérées à l'époque classique :

(7) Outre que les hommes et les femmes pour qui l'amour est banny, j'entends qui n'ont aucune part reelle ou presente en luy ; sont forcez d'advouer, qu'ils y ont part presumptive, ou du moins acceptable, par le mariage : (Gournay, Préface sur les Essais de Michel, seigneur de Montaigne, 1635 : 14-16)

(8) L'instinct qui nous porte à nous agrandir, n'est aucune part si sensible que dans l'ambition : (Vauvenargues, Des lois de l'esprit : florilège philosophique, 1747 : 66-67)

Même si le nombre de ces différentes attestations reste minoritaire (13 occurrences sur un total de 518), il convient de constater que ces cas d'accord se trouvent employés surtout à l'époque moderne (10 occurrences sur un total de 13) :

(9) Contrairement aux allégations de certains journaux, le Général de Gaulle ne prend aucune part directe ou indirecte aux péripéties diverses de l'actuelle crise du régime. (De Gaulle, Discours et messages. 2. Dans l'attente. 1946-1958, $1970: 582-584$ )

\section{Aucune part, variation sémantique}

Le comportement de part comme un «mot-caméléon » est visible, comme nous le verrons, à travers la variation et la richesse sémantique de la construction aucune part: nous avons une intrication des acceptions de part entre, principalement, les notions de «lieu », de «partie/portion d'un tout», et de « participation physique ou morale » à quelque chose, du point de vue lexical. Cette richesse est due à son emploi dans certains types de constructions, à savoir n'avoir aucune part ${ }^{5}$ (304/518) suivie de ne prendre aucune part ${ }^{6}(141 / 518)$. Le reste $(73 / 518)$ est réparti entre ne faire aucune part ${ }^{7}(8 / 518)$, n'être aucune part (5/518), ne laisser aucune part (4/518), ne prétendre aucune part (4/518), ne donner aucune part ${ }^{8}$ (7/518) et d'autres constructions (45/518).

Le tableau ci-contre nous permet d'avoir une vue d'ensemble sur les dates d'apparition et de disparition des principales constructions aux limites, bien évidemment, du corpus étudié (BFM, DMF et Frantext) :

\begin{tabular}{|c|c|}
\hline Constructions & Dates apparition/disparition \\
\hline Ne faire aucune part & $1389-1964$ \\
\hline N'avoir aucune part & $1470-1989$ \\
\hline N'être aucune part & $1481-1761$ \\
\hline Ne prendre aucune part & $1592-1996$ \\
\hline Ne laisser aucune part & $1626-1986$ \\
\hline Ne prétendre aucune part & $1648-1804$ \\
\hline Ne donner aucune part & $1679-1920$ \\
\hline
\end{tabular}

Tableau 1 : Dates d'apparition et de disparition des principales constructions comportant le syntagme aucune part

\subsection{La notion de " lieu »}

La construction aucune part évoque, entre autres, la notion de « lieu » (48/518), qui a subi une évolution diachronique que nous résumons dans le tableau suivant : 


\begin{tabular}{|c|c|c|}
\hline Siècles & $\begin{array}{c}\text { Nombres } \\
\text { d'occurrences }\end{array}$ & $\begin{array}{c}\text { Nombre total } \\
\text { d'occurrences }\end{array}$ \\
\hline $\mathrm{XIII}^{\mathrm{e}}$ & 3 & 3 \\
\hline $\mathrm{XIV}^{\mathrm{e}}$ & 5 & 10 \\
\hline $\mathrm{XV}^{\mathrm{e}}$ & 6 & 9 \\
\hline $\mathrm{XVI}^{\mathrm{e}}$ & 3 & 8 \\
\hline $\mathrm{XVII}^{\mathrm{e}}$ & 9 & 93 \\
\hline $\mathrm{XVIII}^{\mathrm{e}}$ & 9 & 177 \\
\hline $\mathrm{XIX}^{\mathrm{e}}$ & 4 & 101 \\
\hline $\mathrm{XX}^{\mathrm{e}}$ & 9 & 117 \\
\hline
\end{tabular}

Tableau 2 : Aucune part, évolution diachronique de la notion de « lieu »

Nous commençons par évoquer la notion de « lieu » pour la raison suivante : la construction aucune part est apparue pour la première fois au XIII ${ }^{\mathrm{e}}$ siècle, avec une exclusivité spatiale tout au long de ce siècle. Ceci s'expliquerait par le fait que, si on se réfère au Dictionnaire Historique de la Langue Française ${ }^{9}$ (2010 : 1548), part évoque, dès ses premières attestations, une « idée spatiale de «partie d'un espace, point de l'espace », d'abord avec la notion latine de «direction, côté » ». Aucune part évoque le «lieu » dans des constructions positives/négatives avec « quelque part/nulle part» comme équivalents modernes pour renvoyer soit à un lieu concret $\left(\mathrm{du} \mathrm{XIII}{ }^{\mathrm{e}}\right.$ au XVII ${ }^{\mathrm{e}}$ siècle), soit à un lieu abstrait $\left(\mathrm{du} \mathrm{XIV}^{\mathrm{e}}\right.$ au $\mathrm{XX}^{\mathrm{e}}$ siècle), soit à une partie d'un lieu (du XVIII ${ }^{\mathrm{e}}$ au $\mathrm{XX}^{\mathrm{e}}$ siècle). Il convient de noter préalablement qu'il n'est pas toujours aisé de préciser ce sens de «lieu », d'où l'importance de recourir à certains éléments du contexte (sémantisme (verbe de mouvement, vision) ou syntaxe (complément locatif)...) pour lever toute ambiguïté et pouvoir ainsi trancher.

\section{- $\quad$ Lieu concret}

Dans les exemples qui suivent, la notion de « lieu » est repérée grâce à l'emploi des verbes de mouvement sordre $^{10}(10)$, venir (11) et aller (12) accompagnés parfois des prépositions de et en avec les acceptions de « quelque part » :

(10) car il ne porroit estre qu'il ne nos en mescheïst, se guerre nos sordoit d'aucune part, et por ce vos covient il, ausint comme a force, fere nostre volenté de ceste chose. (Mort le Roi Artu, 1230 : 174)

(11) Et quant il venoit de dehors d'aucune part, si lui disoit que il venoit de ribauderie. Si ne le laissoit vivre en pais, ains lui faisoit souffrire et traire la plus dure vie dou monde. (Chronique de Morée, 1320-1324 : 390)

(12) Et commença dès lors enant d'aler sovent veoir, sieuir et acompaignier la dame de Mathegriphon quant elle vouloit aler aucune part. (Ibid., 391)

et de « nulle part »:

(13) Je ne puis maintenant courir Në aller en aulcune part. (La Vigne, Le Mystère de Saint Martin, 1496 : 410-411)

(14) (pour n'oser aller en aucune part divertir son ennuy) (Yver, Le Printemps (extraits), $1572: 1256-1257)$

(15) Que si vous estes celuy que j'imagine, ce bien ne me pouvoit venir d'aucune part, dont il me fust plus cher, (Voiture, Lettres, $1648: 162-164$ ) 
Dans d'autres exemples, seul le contexte permet d'identifier cette notion de «lieu »: idée de vision dans (16-17), complément locatif en cest escript dans (18), complément locatif, en aucune part corrélé au verbe paroitre (paraître) «se montrer » dans (19). Dans (16-18), l'interprétation en " quelque part » est assurée, entre autres, par le contexte forclusif dans lequel se trouve aucune part: après une principale négative (16), dans une interrogation indirecte (17) et dans une proposition hypothétique (18) :

(16) Il ne sont pas iluec a aise C'on nes espit d'aucune part ; Et li vallés d' aus se depart, K' il ne lor velt grever ne nuire. (Renart, Escoufle, entre 1200 et $1202: 109$, v.3373)

(17) lors fist il monter sur le mast pour regarder se on les pourroit nulle part adviser, ne se ilz pourroient veoir terre aucune part. (Berinus T.1, $1350: 38$ )

(18) Se aucune part en cest escript et libelle j'ay erré ou failli, il me soit pardonné, car ce a esté par ignorance. (La Somme Abregiet de Théologie, 1481 : 99)

Dans l'exemple suivant, aucune part a « nulle part » comme équivalent :

(19) Verrina, après l'avoir cherché longtemps en vain, s'étoit remis sur sa galère comme désespéré, parce que les nouvelles qui venoient de tous les quartiers de la ville portoient qu'il ne paroissoit en aucune part. (Retz, La Conjuration du comte Jean-Louis de Fiesque, $1665: 580-581$ )

\section{- Lieu abstrait}

A côté de ce lieu concret, il est question dans les exemples qui suivent d'un lieu abstrait où aucune part a, par exemple, comme équivalent « aucune place » dans une âme :

(20) va donc, encore un coup, va, séducteur infâme : n'espère aucune part désormais en mon âme ; (Corneille, L'Imitation de Jésus-Christ [trad.], 1656 : 294-296)

Cette notion de « lieu vague » est la seule valeur à travers la construction être aucune part (exclusivité : 5 occurrences) :

(21) Dieu est dit estre aucune part par revelation des choses couvertes. (La Somme Abregiet de Théologie, $1481: 138$ )

(22) Cependant il n'y a rien de plus secret que luy, il est par tout, et n'est en aucune part, il se fait sentir, et ne se laisse point toucher ; (Senault, De l'usage des passions, 1641 : 158-160)

dans certains cas à travers laisser... aucune part (1 occurrence sur un total de 4) :

(23) tant y a qu'apres avoir couru diverses contrées deux ans entiers, et n'avoir rien fait que promener vainement son mal sans le pouvoir laisser en aucune part, il se resolut de le raporter au lieu mesme où il l'avoit pris. (Audiguier, Les Amours d'Aristandre et de Cléonice, $1626: 122-125)$

et finalement à travers la construction n'avoir aucune part ${ }^{11}$ (12 occurrences sur un total de 304) avec l'acception de «place » entre le FC (abréviation du français classique) et le FM (français moderne) :

(24) Les abîmes ouverts des célestes rigueurs d'un tremblement égal rempliront tous les cœurs où cette auguste croix ne sera point empreinte ; mais ceux qui maintenant suivent son étendard verront lors tout frémir d'une trop juste crainte, et dans ce vaste effroi n'auront aucune part. (Corneille, L'Imitation de Jésus-Christ [trad.], 1656 : 242-244)

(25) ou peut-être ne voulait-elle pas qu'il existât, et toute sa conversation n'était-elle que pour nier une région de lui dans laquelle elle croyait n'avoir aucune part ; ou peut-être l'aimait-elle moins lorsqu'il était loin. (Moinot, Le Guetteur d'ombre, 1979 : 131-132)

\section{- Partie d'un espace, d'un lieu}

Nous constatons finalement que le lieu peut être exprimé à travers la relation métonymique de «partie d'un lieu » avec par exemple dans (26) l'image d'un arbre généalogique présentant plusieurs côtés. Cette 
idée de «partie d'un lieu » est assurée finalement par la construction ne faire aucune part aux XIX ${ }^{\mathrm{e}}$ et $\mathrm{XX}^{\mathrm{e}}$ siècles (5 occurrences sur 8$)$, une construction qui ne lui est pas exclusive :

(26) Je ne demande qu'une bonne et ancienne noblesse. On sait, madame, que cet avantage ne manque d'aucune part à la vôtre ; et si les conditions, d'ailleurs, étoient agréées mutuellement, je vous avoue que je serois flattée de votre alliance. (Prévost, Nouvelles lettres angloises ou Histoire du chevalier Grandisson [trad.], 1755 : 291-292)

(27) la seconde solution, le soleil semblant destiné à devenir, dans quelques milliards d'années, une étoile géante rouge. Mais on voit l'aléa de ces pronostics, qui ne font aucune part aux accidents / une collision, quoique improbable, n'est pas absolument impossible (Histoire générale des sciences, sous la dir de R. Taton : t. 3 : La Science contemporaine, vol.2 : Le XXe siècle, 1964 : 587)

\subsection{La notion de " partie d'un tout »}

A côté de ce sens de «lieu », aucune part exprime également la notion de «partie d'un tout» (56/518) que nous résumons dans le tableau suivant :

\begin{tabular}{|c|c|c|}
\hline Siècles & $\begin{array}{c}\text { Nombres } \\
\text { d'occurrences }\end{array}$ & $\begin{array}{c}\text { Nombre total } \\
\text { d'occurrences }\end{array}$ \\
\hline $\mathrm{XIV}^{\mathrm{e}}$ & 5 & 10 \\
\hline $\mathrm{XV}^{\mathrm{e}}$ & 3 & 9 \\
\hline $\mathrm{XVI}^{\mathrm{e}}$ & 3 & 8 \\
\hline $\mathrm{XVII}^{\mathrm{e}}$ & 7 & 93 \\
\hline $\mathrm{XVIII}^{\mathrm{e}}$ & 12 & 177 \\
\hline $\mathrm{XIX}^{\mathrm{e}}$ & 9 & 101 \\
\hline $\mathrm{XX}^{\mathrm{e}}$ & 17 & 117 \\
\hline
\end{tabular}

Tableau 3 : Aucune part, évolution diachronique de la notion de «partie d'un tout »

La construction évoque, dans ce sens, une partie d'un ensemble concret ou abstrait.

\section{- Partie d'un tout concret}

Aucune part peut référer à un tout concret $\left(\mathrm{XIV}^{\mathrm{e}}\right.$ et $\mathrm{XVI}^{\mathrm{e}}$ siècles et puis concentration en $\left.\mathrm{FM}\right)$ avec une idée de partage soit de nourriture en MF (28), soit ensuite d'un bien : héritage dans (29-30) :

(28) Et se pouilles, chapons ou cos Ou aucune gourmanderie $\mathrm{Y}$ a, il en a croute ou mie, Et dou seurplus aucune part. (Machaut, La Fonteinne amoureuse, 1361 : 149)

(29) femmes et serviteurs qui se presentent à l'envy à se brusler et enterrer, avec le mary ou maistre trespassé ; loy que les aisnez succedent à tout le bien, et n'est reservé aucune part au puisné, que d'obeissance ; (Montaigne, Essais : t. 1, $1592: 574$ )

(30) J'accepte l'héritage et je n'en repousse aucune part, (Claudel, Le Pain dur, 1918 : 472473)

\section{- Partie d'un tout abstrait}

Aucune part apparaît également dans des constructions évoquant une partie d'un tout abstrait (MF (moyen français) et puis concentration en FM) : 
(31) Et bien croy, se Dieus me gart, Que des dous biens d'amour aucune part Aray de vous, quant vous serez seüre Que je vous aim seur toute creature. (Machaut, La Loange des Dames, 1377 : 173)

(32) Si nous convient deviser comment s'estendra son savoir : c'est qu'elle se sache entendre de toutes choses, car dit le philosophe que cellui n'est pas sage qui ne cognoist aucune part de chascune chose. (De Pizan, Le Livre des Trois Vertus, 1405 : 150)

(33) C'est avec son oeuvre tout entière que nous prierons Dieu ! Rien de ce qu'il a fait n'est vain, rien qui soit étranger à notre salut. C'est elle, sans en oublier aucune part, que nous élèverons dans nos mains connaissantes et humbles. (Claudel, Le Soulier de satin, 1929 : 735-736)

\section{- La notion de « partie d'un tout » et les locutions}

Notons pour finir que le sens de «partie d'un tout » est rendu par un certain nombre de locutions : tout d'abord toutes les attestations concernant la locution prétendre aucune part (4 occurrences) dans des phrases négatives avec sans et ne: des expressions lexicalisées dans la langue classique avec soit un tout abstrait (amitié dans (34)), soit un tout concret aux XVIII et $\mathrm{XIX}^{\mathrm{e}}$ siècles (butin dans (35)) :

(34) Aussi je vous jure, que sans pretendre aucune part en vostre amitié, je me fusse contenté que vous eussiez voulu conserver avec quelque soin, celle que je vous avois voüée, et que vous l'eussiez mise, sinon entre les choses que vous estimiez, au moins entre celles que vous ne voulez pas perdre. (Voiture, Lettres, $1648: 4-6$ )

(35) Les héritiers qui auraient diverti ou recélé des effets d'une succession, sont déchus de la faculté d'y renoncer : ils demeurent héritiers purs et simples, nonobstant leur renonciation, sans pouvoir prétendre aucune part dans les objets divertis ou recélés. (Code civil des Français, 1804 : 144-145)

Nous trouvons ce sens exclusif de «partie d'un tout » également avec la locution ne donner aucune part (7 occurrences) avec un tout concret (partie du butin dans (36)) ou abstrait (partie du gouvernement dans (37), partie de la genèse dans (38)) :

(36) Il punit l'armée consulaire en ne lui donnant aucune part au butin, et obligea Minucius de se démettre du consulat, et de servir dans l'armée en qualité de lieutenant, ce qu'il fit sans plainte et sans murmure. (Rollin, Histoire ancienne des Égyptiens : t.5, $1738: 803$ 805)

(37) Les Romains, trop jaloux de leur autorité, ne donnerent aux femmes aucune part au gouvernement ; (Les Femmes dans la Révolution française : 1789-1794 : t.1 (17891790), $1790: 24-25)$

(38) En effet, les théologiens qui admettent la valeur de la preuve de l'existence de Dieu par le fait de l'obligation, montrent que ce fait entraîne la connaissance certaine de Dieu-en cela vous faites comme eux ; mais ces mêmes théologiens ne donnent à la liberté aucune part dans la genèse du fait de conscience subjectif, qu'on appelle le sentiment de l'obligation. (Dictionnaire de théologie catholique, 1920 : 867)

Citons également la locution ne faire aucune part (3 occurrences sur 8) avec le sens de «partie d'un tout » à partager soit concrètement (39), soit par la parole (partie de mes secrets) d'où la traduction de « révéler, informer » $(40)$ :

(39) le chevalier dist que la terre qu'il tenoit estoit de son conquest, si n'estoit tenus oultre son gré en faire aucune part à son filz, qui contre lui avoit forfait, s'il ne lui plaisoit, et meismes après sa mort, pour ses desmerites, le desheriteroit. (De Pizan, Le Livre Des Fais et Bonnes Meurs du Sage Roy Charles V, 1404 : 72)

(40) Je ne répondis point à ce discours, quoique j'eusse autant d'expérience qu'elle sur cet article ; mais quelque penchant que j'eusse pour Lindamine, j'étois retenuë sur ce qui me regardoit, et je ne lui avois fait encore aucune part de mes secrets ; (Mouhy, La Paysanne parvenue ou les Mémoires de Mme la Marquise de L. V., 1735 : 425-427)

Ce sens est assuré aussi par la locution ne laisser aucune part (2 occurrences sur 4) : 
(41) Il est donc tenté d'utiliser au maximum possible les matériaux ne nécessitant aucun conditionnement préalable et dont la tarification précise ne laisse aucune part d'aléa dans l'établissement de son prix de vente. (L'Industrie française du bois, $1955: 36$ )

(42) c'était dans une haine commune que les deux rivaux se rencontraient envers ledit représentant d'un État, qui ne leur laissait aucune part dans leur immortelle découverte. (Verne, La Chasse au météore, $1986: 238-240$ )

Comme énoncé dans le point précédent, la locution n'avoir aucune part est utilisée pour rendre compte principalement de la notion de "participation». Elle peut évoquer, néanmoins, la notion de «lieu» comme c'est le cas dans les exemples déjà cités (13-31) et également celle de «partie d'un tout » (19 occurrences sur un total de 304) soit concret (43) entre le XVI ${ }^{\mathrm{e}}$ et le XVIII ${ }^{\mathrm{e}}$ siècle, soit abstrait (44) entre le $\mathrm{XIV}^{\mathrm{e}}$ et le $\mathrm{XX}^{\mathrm{e}}$ siècle :

(43) L'éducation des fils, ai-je continué, ne regarde que moi, comme celle des filles appartient à la mère. Je consens que le bien d'Italie soit le partage des filles, et qu'elles soient élevées sous vos yeux. Les fils n'y auront aucune part. (Prévost, Nouvelles lettres angloises ou Histoire du chevalier Grandisson [trad.], 1755 : 206-207)

(44) Le fils aîné acquitte la dette envers les ancêtres, il doit donc tout avoir. " le droit grec est issu des mêmes croyances religieuses que le droit hindou ; il n'est donc pas étonnant d'y trouver aussi, à l'origine, le droit d'aînesse. Sparte le conserva plus longtemps que les autres villes grecques, parce qu'elle fut plus longtemps fidèle aux vieilles institutions ; chez elle le patrimoine était indivisible et le cadet n'avait aucune part. (Fustel de Coulanges, La Cité antique, 1864 : 98-99)

Remarquons finalement que la locution ne prendre aucune part qui, nous le verrons dans le point suivant, évoque la notion de "participation », est utilisée dans deux cas pour exprimer celle de " partie d'un tout » dans le cadre d'un héritage :

(45) Ce principe est, que l'héritier ou le conjoint survivant qui soustrait ou recèle des effets de la succession, doit les rendre en totalité sans pouvoir y prendre aucune part : tout appartient à l'héritier ou au conjoint au préjudice duquel les objets ont été divertis, ou recelés. (Simon, Les Géorgiques, $1981: 451-452$ )

\subsection{La notion de « participation »}

Aucune part se trouve employé finalement pour rendre compte de la notion de « participation » d'abord minoritairement à travers les locutions ne laisser aucune part (1 occurrence sur 4) et ne s'attribuer aucune part (1 occurrence) : participation à une action, à un fait dans des tournures négatives :

(46) Et n'y a t-il pas de l'impieté à vouloir persuader que le secours des dieux deshonore les heros ? Car il les deshonoreroit certainement, si ce secours faisoit tout, et ne laissoit aucune part à leur courage. (Dacier, Des causes de la corruption du goust, 1714 : 127)

(47) Mais son chagrin n'était pas mélangé de remords. Il ne s'attribuait aucune part dans l'accident qu'il déplorait plus que personne. (Gautier, Récits fantastiques, 1856 : 386)

Cette notion de «participation » est rendue essentiellement par deux locutions à savoir ne prendre aucune part (139 occurrences sur un total de 141) et n'avoir aucune part (273 sur un total de 304 occurrences) que nous résumons dans le tableau suivant :

\begin{tabular}{|c|c|c|}
\hline Siècles & Ne prendre aucune part & N'avoir aucune part \\
\hline $\mathrm{XVI}^{\mathrm{e}}$ & 0 & 2 \\
\hline $\mathrm{XVII}^{\mathrm{e}}$ & 13 & 64 \\
\hline $\mathrm{XVIII}^{\mathrm{e}}$ & 37 & 118 \\
\hline
\end{tabular}




\begin{tabular}{|c|c|c|}
\hline Siècles & Ne prendre aucune part & N'avoir aucune part \\
\hline $\mathrm{XIX}^{\mathrm{e}}$ & 45 & 42 \\
\hline $\mathrm{XX}^{\mathrm{e}}$ & 44 & 47 \\
\hline
\end{tabular}

Tableau 4 : Ne prendre/n'avoir aucune part, évolution diachronique de la notion de «participation »

La locution prendre aucune part est utilisée dans des phrases négatives, pour exprimer une participation soit à une sensation/un sentiment, soit à un acte de parole/écriture, soit à une action, de manière passive ou active. C'est d'ailleurs le cas, comme nous l'avons vu précédemment, du versant positif prendre part évoquant une «participation physique ou morale à quelque chose ».

\section{- Participation à une sensation/un sentiment}

C'est le cas de 11 attestations sur un total de 139 occurrences que l'on trouve réparties entre le FC et le FM :

(48) Ne prens aucune part, aux maux que j'ay souffers, Et songe que le sort vient de rompre tes fers. (Scudéry, Arminius ou les Frères ennemis, 1644 : 21-23)

(49) Nous sommes en carnaval. Il y a pourtant des gens qui s'amusent ! La nature ne prend aucune part à ma détresse. (Hugo, Mille francs de récompense, $1866: 691$ )

(50) Ils admirent la délicate harmonie des nombres et des formes ; ils s'émerveillent quand une découverte nouvelle leur ouvre une perspective inattendue ; et la joie qu'ils éprouvent ainsi n'a-t-elle pas le caractère esthétique, bien que les sens n'y prennent aucune part? (Poincaré, La Valeur de la science, 1905 : 138-140)

\section{- Participation par le biais de la parole/l'écriture}

C'est le cas de 19 occurrences sur un total de 139 attestations réparties entre le FC et le FM :

(51) Comme il avait hautement improuvé le violent procédé de Leurs Excellences, il crut, dans sa générosité, me devoir un témoignage public qu'il n'y prenait aucune part, et ne craignit pas de sortir de son bailliage pour venir me faire une visite à Bienne. (Rousseau, Les Confessions, 1778 : 773-774)

(52) Arnauld De Villeneuve mourut dans un naufrage en vue de Gênes, où il fut enseveli en I 3 i 3. On lui attribue quantité d'ouvrages auxquels il ne prit aucune part. Quant à ceux qu'il écrivit réellement, le grand inquisiteur de Tarragone les fit brûler, pour cause d'hérésie, trois ans après sa mort. (Caron \& Hutin, Les Alchimistes, 1959 : 32-33)

\section{- Participation sans intérêt/sans réagir}

Nous avons repéré 16 attestations sur un total de 139 évoquant une participation sans porter d'intérêt ou de réaction et ceci entre le $\mathrm{XVIII}^{\mathrm{e}}$ et le $\mathrm{XX}^{\mathrm{e}}$ siècle. La participation sans intérêt se trouve employée par exemple dans le cadre d'un rituel religieux (la personne ne participe pas entièrement au rituel : dit la messe mais ne s'y investit pas dans (53)) ou social (apporter une collation de fruits dans (54) ou faire des dons dans (55)) :

(53) qu'on aprit qu'avec un peu d'adresse, sans crime un prêtre peut vendre trois fois sa messe ; pourvû que, laissant là son salut à l' écart, lui-même en la disant n'y prenne aucune part. (Boileau-Despréaux, Satire XII sur l'équivoque, 1711 : 222-224)

(54) On aporta une collation composée de fruit de limonades et de confitures seches. L'inconnu n'y prit d'abord aucune part, ensuite imaginant qu'il tenoit une plume au lieu d'un crayon il la trempa dans le verre de limonade qu'il prenoit pour un encrier. (Potocki, Manuscrit trouvé à Saragosse, 1815)

(55) Elle pratiquait, mais sans amour, par conséquent sans foi vivante ; elle donnait de l'argent pour des messes, mais elle ne prenait aucune part active à des oeuvres ; toute 
sa religion reposait sur cette pensée unique : la revoir ! Le reste, que lui importait ?

(Rolland, Jean-Christophe : Dans la maison, 1909 : 1055-1056)

\section{- Participation à une action}

C'est le cas de 93 attestations sur un total de 139 occurrences que l'on trouve réparties entre le FC et le FM. Elles évoquent principalement une action guerrière dans (56), de divertissement dans (57), criminelle dans (58) et politique dans (59) :

(56) On verra en premier lieu par ce récit, que des cités renfermées dans les provinces obéïssantes ne prirent aucune part à la guerre de Syagrius contre Clovis : elles ne tirerent point l'épée pour deffendre ce romain. (Dubos, Histoire critique de l'établissement de la monarchie françoise dans les Gaules, 1734 : 7-8)

(57) Lorsque le taureau n'est pas mort sur le coup, on voit sauter par-dessus la barrière un petit être mystérieux, vêtu de noir, et qui n'a pris aucune part à la course : c'est le cachetero. I (Gautier, Voyage en Espagne, 1843 : 120-121)

(58) Mais n'est-il pas providentiel de voir des hommes, après longues années, les uns démontrer l'irrégularité d'un meurtre auquel ils n'avaient pris aucune part, les autres accourir, sans qu'on le leur demandât, devant l'accusation publique ? (Chateaubriand, Mémoires d'outre-tombe : t.2, $1848: 146-149)$

(59) Profitant de son indifférence, les partis truquèrent le scrutin. Vous savez ce qui est advenu. Les choses étant ce qu'elles sont, j'ai décidé, depuis deux ans, de ne prendre aucune part à ce qui se passe à l'intérieur du régime. (De Gaulle, Discours et messages. 2. Dans l'attente. 1946-1958, $1970: 627-628)$

La seconde locution n'avoir aucune part évoque, comme c'est d'ailleurs le cas actuellement de la locution avoir part vue précédemment, une «participation» soit morale soit physique. Nous rajoutons un troisième cas concernant une participation par le biais de la parole.

\section{- Participation morale}

Citons principalement des participations à des sensations ou sentiments entre le FC et le FM :

(60) Les yeux, les oreilles, les narines et la langue peuvent recevoir des impressions qui ne dépendent que du toucher seul, et d'où naissent des douleurs auxquelles ni les couleurs, ni les sons, ni les odeurs, ni le goût n'ont aucune part. (Bossuet, De la connaissance de Dieu et de soi-même, 1704 : 94-95)

(61) Cette femme, il ne la connaît pas, elle a des plaisirs, des chagrins, des amours où il n'a aucune part. Il n'existe pas pour elle, et peut-être, s'il lui parlait, se moquerait-elle de lui comme Marguerite avait fait de moi. (Dumas, La Dame aux camélias, 1848 : 128129)

\section{- Participation physique}

La participation physique regroupe, quant à elle, la majorité des attestations repérées entre le FC et le FM. Elle évoque essentiellement une participation en tant que cause à des actions soit criminelles dans (62), soit politiques dans (63), soit en rapport avec la religion dans (64) :

(62) mais cette poursuite passa toutes bornes de justice, dautant qu'au decret de la condemnation on comprit non seulement les coulpables, mais aussi plusieurs de ceux qui mesmes n'estoient pas à Rome quand le meurtre fut fait, et entr'autres le jeune Pompée, auquel on ne pouvoit reprocher qu'il eust eu aucune part à ce crime ; (Coëffeteau, Histoire romaine, $1646: 130)$

(63) Les intendans des provinces, tous les juges, tous les magistrats, et même ceux de toutes les plus grandes et plus considerables villes du roiaume qui n'ont plus aucune part dans le gouvernement de l'etat, (Meslier, Mémoire des pensées et des sentiments : $t .3$ [Preuve 8 et conclusion], 1729 : 136-138) 
(64) Ayant la religion de la cité, il pouvait en invoquer la loi et accomplir tous les rites de la procédure. L'étranger au contraire n'ayant aucune part à la religion n'avait aucun droit. (Fustel de Coulanges, La Cité antique, $1864: 248-250$ )

Elle évoque également une participation à une action décrite comme le produit d'une chose et non pas d'une autre comme dans (65-67) (l'âme des tragédies comme le produit de la grandeur de courage et non pas de la tendresse et des passions dans (65)) ou comme le produit d'une chose plus qu'une autre (la révolution est le produit de ce ressentiment bien plus que l'enthousiasme dans (66)) :

(65) La tendresse et les passions, qui doivent être l'âme des tragédies, n'ont aucune part en celle-ci : la grandeur de courage y règne seule, et regarde son malheur d'un oeil si dédaigneux qu'il n'en sauroit arracher une plainte. (Corneille, Nicomède : Au lecteur, $1651: 501-502)$

(66) Ce ressentiment, nourri dans tous les coeurs, fut le vrai motif de la révolution, bien plus que l'enthousiasme, qui n'y eut aucune part ; il anime encore la nation après deux siecles entiers ; (Linguet, Histoire impartiale des Jésuites, $1768: 133-135$ )

(67) L'observation de ce qui se passe à cet égard, doit nous montrer qu'il est plus juste de dire : que les raisonnemens, et que les déterminations qui sont la suite de jugemens, prennent leur source dans des opérations de l'intelligence ; tandis que l'instinct qui fait exécuter quelqu'action, prend la sienne dans des besoins et des penchans qui émeuvent immédiatement le sentiment intérieur de l'individu, et le font agir sans choix, sans délibération, en un mot, sans que l'intelligence y ait aucune part. (Lamarck, Philosophie zoologique, 1809 : 447-449)

\section{- Participation par le biais de la parole}

(68) Et certes il y a bien de l'apparence, que par une particuliere eslection cette personne ait esté choisie pour recevoir la paix, qui la recevra dans des mains nettes de toute sorte d'injustice ; avec un esprit vuide de toute l'aigreur et de toute l'animosité des partis ; pur et innocent de toute la violence des choses passées : qui n'a eu aucune part à aucun mauvais conseil. (Balzac, Dissertations politiques, 1654 : 475-476)

(69) Que si M Goëzman prétend nier la liberté qu'il s'est donnée sur une déclaration à laquelle il dit n'avoir aucune part, (Beaumarchais, Mémoires contre M. Goëzmann, $1774: 101-103)$

\section{Aucune part, un cas de grammaticalisation ? de lexicalisation ?}

L'étude des caractéristiques sémantiques de aucune part corrélée à celle de ses caractéristiques morphosyntaxiques vues dans le point (1) nous permettront de nous interroger ici sur la nature de son changement linguistique : s'agit-il d'un cas de grammaticalisation ou de lexicalisation ? Pour essayer d'y répondre, nous considérerons aussi bien l'emploi de aucune part comme locution adverbiale au sens spatial de «quelque part »/«nulle part » et son emploi avec des verbes supports ${ }^{12}$ (avoir part, prendre part...).

\subsection{Aucune part, un cas de grammaticalisation?}

Avant de répondre à cette question, il convient de rappeler rapidement la définition la plus courante de cette notion :

Under the diachronic aspect, grammaticalization is a process which turns lexemes into grammatical formatives and makes grammatical formatives still more grammatical (cf. Kuryłowicz 1965:52). (Lehmann, $1985: 303$ )

En linguistique diachronique, on parle de grammaticalisation quand un morphème lexical, au cours de l'évolution d'une langue ou dans la transformation d'une langue à une autre, est devenu un morphème grammatical. (Dictionnaire de linguistique, 1994, nouvelle édition 1999 : 226 in Bertrand, 2007 : 42) 


\begin{abstract}
The derivational pattern which the word grammaticalization belongs to suggests that it means a process in which somethig become or is made grammatical. In view of this, the term is unfortunate in several respects [...] Secondly, in addition to the above explication, grammaticalization must mean a process in which something becomes or is made more grammatical. (Lehmann, 1995 : 9 in Bertrand, 2007 : 42)
\end{abstract}

Grammaticalization [...] is that subset of linguistic changes whereby lexical material in highly constrained pragmatic and morphosyntactic contexts becomes grammatical, and grammatical material becomes more grammatical. (Traugott, $1996: 183$ )

Le terme de "grammaticalisation » désigne le processus linguistique par lequel des unités lexicales se convertissent en morphèmes grammaticaux. (De Mulder, 2001 : 8)

processus, qui consiste en l'évolution d'une forme lexicale vers un statut grammatical, ou en celle d'une forme grammaticale vers un statut plus grammatical. (Prévost, $2006: 122)$

évolution d'une forme lexicale vers une forme grammaticale (ou évolution d'une forme grammaticale vers une forme plus grammaticale). (Prévost \& Fagard, 2007 : 34)

Ces différentes définitions présentent un point commun résidant, comme le note Bertrand (2007: 42) à propos des cinq définitions ${ }^{13}$ qu'il retient concernant la grammaticalisation, dans le « mouvement général, de dynamisme » qui se résume dans le passage d'une forme lexicale vers une forme grammaticale ou d'une forme grammaticale vers une forme plus grammaticale : processus par lequel un mot lexical ou un groupe de mots perd l'intégralité ou une partie de son sens lexical pour remplir une fonction plus grammaticale. Cette définition de la grammaticalisation, faisant intervenir unités lexicales et unités grammaticales, nécessite de clarifier ce qui peut être considéré comme lexical et ce qui peut être grammatical, en d'autres termes ce qui relève du lexique ou de la grammaire. Où se situe, comme s'interroge Prévost (2006 : 133), « la frontière entre catégories lexicales et grammaticales, ou, formulé en d'autres termes, entre catégories majeures et mineures, entre classes ouvertes et fermées »? Elle note (Ibid., 133-134) que «beaucoup admettent l'idée d'un continuum entre classes, plutôt que celle d'une frontière nette. » Nous citons, en l'occurrence, Marchello-Nizia (2006:20) :

Dès lors qu'il est possible pour un mot de passer du lexique à la grammaire, et spécialement des catégories majeures (nom, verbe) à des catégories secondaires (adverbes, prépositions) dont certaines ont même perdu leur autonomie et sont devenues des composants de mot (affixes), il faut poser que la frontière entre les différentes sortes d'unités n'est pas absolue. Il s'agit d'un continuum, sur lequel on peut situer des étapes dans le passage d'une catégorie à l'autre, et définir une 'échelle' allant du plus lexical au plus grammatical. [lexique/grammaire/plus lexical/plus grammatical].

Prévost (Ibid., 134) rajoute que si «certaines catégories se placent assez facilement du côté lexical (les noms par exemple) ou grammaticales (les conjonctions), d'autres sont plus difficiles à étiqueter » comme les adverbes (ne est considéré comme grammatical alors que les adverbes de manière en -ment sont plutôt lexicaux) ou les verbes (l'auxiliaire « est-il « lexical » au même titre qu'un verbe plein, ou est-il plutôt «grammatical »? Faut-il créer deux catégories ? »). Cette distinction en unités lexicales et grammaticales « devient plus difficile encore lorsque l'on considère les expressions complexes. Le fait qu'elles soient composées de formes lexicales rend moins évident leur classement du côté « grammatical » (Ibid.)

L'étude des caractéristiques morphosyntaxiques de aucune part nous a permis de constater une réduction de la liberté de construction de ces dernières dans la mesure où nous assistons à la disparition du pluriel $\left(\mathrm{XIV}^{\mathrm{e}}\right.$ siècle) et des cas d'insertion (XVII ${ }^{\mathrm{e}}$ siècle), deux arguments en faveur de l'idée de sa grammaticalisation si nous suivons le raisonnement de Lehmann (1985: 306) observant que l'un des critères de la grammaticalisation consiste dans le fait que plus une forme se grammaticalise, moins elle a de liberté :

It is set up according to a set of criteria which concern the autonomy of the language sign. The more freedom with which a sign is used, the more autonomous it is. The grammaticalization of a sign detracts from its autonomy. Consequently, if we want to 
measure the degree to which a sign is grammaticalized, we will determine its degree of autonomy.

On doit cependant tenir compte du fait que ces cas sont très minoritaires (4 occurrences sur un total de 518).

Les cas de reprise en anaphore, de construction directe et de caractérisation adjectivale permettent de constater, en revanche, que aucune part ne peut pas être considéré comme un cas de grammaticalisation, contrairement à certaines constructions ${ }^{14}$, en l'occurrence quelque part où part selon Blanche-Benveniste (2003 : 286) ne peut plus s'identifier comme un «élément nominal»; il n'est pas possible de lui adjoindre, même via la préposition de, des modifieurs, comme on peut le faire pour quelqu'un et quelque chose (quelqu'un de bien, quelque chose de beau) :

(70) * Je veux aller quelque part de beau. (Blanche-Benveniste, $2003: 286$ )

Elle rajoute qu'il a « en quelque sorte l'exclusivité de la valeur indéfinie pour le lieu, puisque les autres termes comme endroit, lieu, côté, coin, ne lui font plus concurrence » :

(71) Où est-il ?- quelque part. (Ibid.)

(72) Où est-il ? - ? en quelque lieu, en quelque endroit, en quelque coin. (Ibid.)

L'étude de la notion de « lieu », représentée actuellement, comme le constate Blanche-Benveniste (2003), par quelque part, nous amène à nous demander si la construction aucune part peut l'être également. Nous considérons que aucune part en emploi spatial peut être considéré, dans certaines circonstances, comme un cas de grammaticalisation. Il désigne dans le $D M F$ une localisation indéfinie avec l'acception de «à un endroit indéterminé », produisant un adverbial indéfini, dont les termes connaissent le même degré de figement que ceux de quelque part. Dans les phrases négatives, la construction pourrait se rendre par «en aucun lieu ». Ce sens de lieu est utilisé jusqu'à nos jours de manière assez fréquente en français parlé à l'île Maurice, et plus encore en créole mauricien à travers l'expression okenn par. Nous donnons l'exemple de ces différents échanges ${ }^{15}$, publiés le 1 juillet 2011 , autour de la question suivante : est-ce que « aucune part est une expression recevable en «bon» français ? ». Cette question a été posée suite à l'anecdote suivante : en cherchant un étui d'appareil photo qui paraissait introuvable, une personne a dit en français : "Je ne sais pas où il est; je le trouve aucune part. ", comme si aucune part était l'équivalent du français « nulle part». Cette question de l'emploi ou pas de aucune part en bon français a tellement interpelé le déclencheur de ce forum qu'il s'est surpris à dire, en cherchant désespérément le bouchon du lavabo dans certaines parties de la maison, "il n'y a aucun bouchon aucune part ». Les différents internautes se mettent d'accord sur le fait que cette expression ne se trouve plus actuellement en usage pour le lieu, qui est rendu plutôt par l'expression « nulle part ». Et on déduit alors que aucune part serait un mauricianisme - avec le sens de «nulle part», « en aucun lieu» - qui « rimerait avec archaïsme ». En plus de cet emploi spatial de aucune part en créole mauricien, nous constatons que cette construction indiquant un lieu abstrait ou métonymique continue à s'employer en FM, ce qui va à l'encontre de ce qu'avancent Arrivé et al. (1986: 327) qui, semble-t-il, parlent d'un lieu concret: «aucune part n'est pas attesté dans le sens local dans la langue moderne ». Rien ne nous empêche de considérer alors aucune part comme un cas de grammaticalisation en ce qui concerne le lieu dans la mesure où nous avons une évolution progressive et continue d'un lieu concret vers un lieu abstrait ou métonymique. Par ailleurs, quelque part, considéré comme un cas de grammaticalisation avec une exclusivité de la notion de « lieu », peut être employé avec la valeur de « en quelque sorte »:

\section{(73) On se moque d'elle, quelque part.}

Nous avons trouvé également des exemples où part dans quelque part n'est pas grammaticalisé. En témoignent ces cas de pluriel dans (75) et d'insertion d'indéfini (autre) dans (76) ou d'adjectif (immense) dans (77) entre quelque et part:

(74) Il n'avait recueilli de l'opulence paternelle que quelques parts de la raffinerie de Maestricht dont les revenus trouvaient rarement le chemin de sa poche, et une des moindres terres familiales, (Yourcenar, L'Oeuvre au noir, $1968: 659-660$ ) 
(75) Au bout de l'année, il vous faudrait toujours en louer un quelque autre part. (Sand, $L a$ Petite Fadette, 1849 : 54-55)

(76) Car il doit incarner le bien commun... " la science politique intégrale, quelque immense part qu'il convienne d'y réserver à l'observation et à l'induction, est non seulement d'un ordre supérieur à la science inductive, (Maritain, Primauté du spirituel, 1927 : 189190)

L'étude de la grammaticalisation de aucune part nous amène à la conclusion suivante : réticence à la grammaticalisation de part dans aucune part (on ne peut décider de la catégorie grammaticale à laquelle appartiendrait le nouvel aucune part 'grammaticalisé') et même dans quelque part. D'où la nécessité de chercher une autre solution résidant peut-être dans la lexicalisation de aucune part.

\subsection{Aucune part, un cas de lexicalisation?}

Avant de répondre à cette question, il convient préalablement de définir cette notion. Evoquée le plus souvent en rapport avec la grammaticalisation, la lexicalisation, comme le note Prévost (2006: 128) « jouit néanmoins d'une certaine autonomie, et correspond d'ailleurs à des concepts et à des approches assez variés. » D’après Marchello-Nizia (2006 : 99), c'est Kurylowicz qui a introduit le mot lexicalisation "pour désigner le phénomène de passage d'une unité de la grammaire au lexique. " Dans le même contexte, Brinton \& Traugott (2005 : 96) cités par Fagard \& De Mulder (2007 : 26) lui donnent la définition suivante :

\section{Lexicalization is the change whereby in certain linguistic contexts speakers use a syntactic construction or word formation as a new contentful form with formal and semantic properties that are no completely derivable or predictable from the constituents of the construction or the word formation pattern.}

Marchello-Nizia (Ibid.) utilise ce terme de lexicalisation, « dans un sens bien plus large, pour désigner l'introduction dans le lexique de nouveaux termes, par quelque moyen que ce soit, et à quelque fin que ce soit, en particulier pour répondre à un besoin social. " Bref, considérant l'étude de Wischer (2000:358), les linguistes ne sont pas toujours d'accord sur l'utilisation du terme de lexicalisation. Certains la décrivent comme un processus permettant de transformer des groupes syntaxiques libres ou des formations ad hoc en unités lexicales en leur ajoutant une composante sémantique spécifique :

lexicalization as the process that turns free syntactic groups or ad-hoc-formations into lexical units by adding a specific semantic component, thus identifying it with idiomaticization, others consider idiomaticization a consequence of lexicalization.

D'autres la considèrent comme le transfert de toute forme linguistique dans le lexique d'une langue. Selon cette approche, le développement de tout nouveau lexème est considéré comme un cas de lexicalisation.

Tenant compte de ces différentes définitions, nous nous demandons si aucune part à travers les locutions verbales n'avoir aucune part (304 occurrences), ne donner aucune part (7 occurrences), n'être aucune part (5 occurrences), ne faire aucune part (8 occurrences), ne laisser aucune part (4 occurrences), ne prendre aucune part (141 occurrences) et ne prétendre aucune part (4 occurrences) constitue une structure figée et si ce figement relève de la lexicalisation ou pas. Avant cela, nous commençons par définir la notion de locution verbale figée : «Une locution verbale s'oppose à une suite libre verbe + complément, c'est-à-dire qui n'est contrainte que par le domaine d'arguments du verbe et qui a toutes les transformations potentielles. » (Gross, 1996 : 70 in Bertrand 2007 : 43) Elle présente les caractéristiques suivantes :

- Les locutions verbales ont la même structure interne que les groupes verbaux libres ; elles peuvent avoir formellement un complément direct (prendre la tangente) ou indirect (...prendre le taureau par les cornes) ; - les locutions verbales comportent toujours une forme verbale susceptibles d'être fléchie (conjuguée) ; - les locutions verbales ne sont pas toutes figées au même degré, de sorte qu'il y a un continuum entre les groupes verbaux libres et les locutions verbales entièrement figées. 


\section{L'indication du degré de figement se reflète dans les possibilités transformationnelles. (Ibid., 44)}

Les différentes locutions comprenant aucune part connaissent un figement sémantique (opacité sémantique) dans la mesure où, comme vu précédemment, la notion de «lieu» (49/518) est rendue principalement par les constructions avoir aucune part (12/304), être aucune part (5/5), laisser aucune part (2/4) et faire aucune part (5/8) ; la notion de "partie d'un tout » (55/518) est rendue essentiellement par avoir aucune part (19/304), prendre aucune part (2/141), laisser aucune part (1/4), prétendre aucune part (4/4), donner aucune part (7/7), et faire aucune part (3/8); la notion de «participation » (414/518) finalement se trouve rendue par avoir aucune part (273/304), prendre aucune part (139/141) et laisser aucune part (1/4).

Elles présentent en outre un figement syntaxique. Prenons l'exemple de la locution prendre aucune part et essayons de lui appliquer certains des tests utilisés par Bertrand (Ibid.) sur prendre congé et chercher noise pour montrer «le caractère contraint» de ces structures syntaxiques : extraction, passif et pronominalisation :

(77) Pascal n'a pris aucune part au meurtre. (Reformulation de l'exemple (58))

(78) *Ce n'est aucune part au meurtre que Pascal a pris. / *Aucune part au meurtre n'a été prise par Pascal. / *Pascal ne l'a prise, aucune part au meurtre. ${ }^{16}$

Ces différents tests montrent que la structure syntaxique du syntagme prendre aucune part est contrainte, ce qui plaide en faveur de son figement. Ce figement syntaxique correspond selon les termes de Bertrand (Ibid.) « à une cristallisation de la structure interne au syntagme ». Pour résumer, l'exemple (78) constitue une structure figée syntaxiquement (cristallisation) et sémantiquement («participation»). Bertrand rajoute (Ibid.) que les différentes formes infinitives ou conjuguées de prendre congé ne présentent aucun changement au niveau de l' " appartenance à la classe grammaticale d'origine » dans la mesure où «le verbe reste un verbe et le complément demeure un substantif. »C'est d'ailleurs le cas de prendre aucune part : à «partir du moment où les locuteurs ont compris le syntagme comme une seule entité insécable sémantiquement, cette structure s'est figée syntaxiquement. » (Ibid., 46) Bertrand (2007 : 50) conclut que les «locutions verbales sans changement de classe grammaticale» ne peuvent pas être considérées comme des cas de grammaticalisation dans la mesure où «le changement opéré ne touche que la grammaire de surface ». Ceci relève plutôt, comme c'est le cas des locutions figées autour de aucune part, du domaine de la lexicalisation.

\section{Conclusion}

Cette étude nous a permis de montrer, à partir des caractéristiques morphosyntaxiques et sémantiques de aucune part, que cette construction est complexe dans la mesure où certains critères plaideraient en faveur de sa grammaticalisation alors que d'autres non. Son apparition dans des locutions verbales figées comme avoir aucune part et prendre aucune part évoquant par exemple la notion de «participation» constitue un indice de sa lexicalisation. Il serait intéressant d'étudier une autre piste concernant la nature du changement linguistique de aucune part dans sa globalité dans la mesure où nous avons le passage d'une structure considérée comme un bloc lorsqu'elle a les sens de «quelque part» ou de " nulle part» vers une structure analysable en déterminant + substantif, ce qui plaiderait semble-t-il en faveur de sa dégrammaticalisation : passage d'une forme plus grammaticale vers une forme moins grammaticale.

\section{Références bibliographiques}

Arrivé, M., Gadet, F. \& Galmiche, M. (1986). La grammaire d'aujourd'hui : guide alphabétique de linguistique française. Paris : Flammarion.

Bertrand, O. (2007). Locutions figées sans changement de classe grammaticale. In Combettes, B. \& Marchello-Nizia, C. (éd.), Etudes sur le changement linguistique en français, Nancy : Presses Universitaires de Nancy, 41-52.

Blanche-Benveniste, C. (2003). Quelqu'un, quelque chose, quelque part, quelquefois. Verbum, 25/3, 277-290. 
De Mulder, W. (2001). La linguistique diachronique, les études sur la grammaticalisation et la sémantique du prototype : présentation. Langue française, 130, 8-32.

Dictionnaire du Moyen Français (1330-1500). http://www.cnrtl.fr/definition/dmf/

Fagard, B. \& De Mulder, W. (2007). La formation des prépositions complexes : grammaticalisation ou lexicalisation ?. Langue française, 156/4, 9-29.

Godefroy Dictionnaire, Dictionnaire de l'ancienne langue française et de tous ses dialectes du IX au XV siècle. http://micmap.org/dicfro/search/dictionnaire-godefroy

Godefroy Complément, Dictionnaire de l'ancienne langue française et de tous ses dialectes du IXe au $X V^{e}$ siècle. Complément. http://micmap.org/dicfro/search/complement-godefroy

Huguet, E. (1925-1967). Dictionnaire de la langue française du seizième siècle. Paris: Edouard Champion. http://www.classiques-garnier.com.faraway.u-paris10.fr/numerique-bases/

Lehmann, Ch. (1985). Grammaticalization : synchronic variation and diachronic change. Lingua e Stile, 20, 303-318.

Littré, E. (1963). Le Dictionnaire de la langue française http://ittre.reverso.net/dictionnaire-francais/definition/

Marchello-Nizia, Ch. (2006). Grammaticalisation et changement linguistique. Bruxelles : De Boeck.

Prévost, S. (2006). Grammaticalisation, lexicalisation et dégrammaticalisation : des relations complexes. Cahiers de praxématique, $46,121-146$.

Prévost, S. \& Fagard, B. (2007). Grammaticalisation et lexicalisation : la formation d'expressions complexes. Langue française, 156/4, 3-8.

Rey, A., Tomi, M., Hordé, T. \& Tanet, Ch. (2010). Dictionnaire Historique de la Langue Française. Le Robert.

Traugott, E. C. (1996). Grammaticalization and lexicalization. In Brown, K. \& Miller, J. (éd.), Concise Encyclopedia of Syntactic Theories. Oxford: Pergamon, 181-187.

Trésor de la Langue Française informatisé, Dictionnaire de la langue française des XIX et XXe siècles. http://www.cnrtl.fr/definition/

Wischer, I. (2000). Grammaticalization versus lexicalization 'Methinks' there is some confusion. In Fischer, O., Rosenbach, A. \& Stein, D. (éd.), Pathways of Change : Grammaticalization in English. USA : John Benjamins Publishing Co., 355-370.

\footnotetext{
${ }^{1}$ Nous avons consulté la version électronique de ces dictionnaires.

2 http://bfm.ens-lsh.fr/.

3 http://www.atilf.fr/dmf/.

${ }^{4}$ http://www.frantext.fr.

${ }^{5}$ Le versant positif avoir part est utilisé essentiellement pour exprimer les notions de «partie d'un tout » $(D M F)$, de "participation morale », ce qui relève de l'abstrait ( $G D$ : porter un intérêt, $D M F, D L$ et $T L F i)$ ou "participation physique » relevant du concret ( $D M F, D L$ et $T L F i)$ et de « partie d'un tout » (concret dans $D M F$ et abstrait dans $D L$ ).

${ }^{6}$ Le versant positif prendre part est utilisé dans le $D L$ et le $T L F i$ pour exprimer deux notions : "participation concrète à quelque chose» (part aux jeux) et «participation morale» (part d'affection, d'amour: partager une circonstance joyeuse ou douloureuse).

${ }^{7}$ Avec le versant positif faire part, nous passons de la notion de «partie d'un tout concret » (DMF, DH et $D L$ : partage équitable ou inéquitable de quelque chose de concret), à celle de «partie d'un tout abstrait» $(D L:$ partie du pouvoir et $T L F i$ : réserver une place abstraite), à celle de «tenir en compte » (DL et $T L F i)$, à celle de " information » $(D L$ et $T L F i)$ et à celle de « ce qui revient à » $(T L F i)$.

${ }^{8}$ En ce qui concerne la construction positive donner part, nous passons de la notion de «lui faire un mauvais sort » $(D M F)$ à celles de « participation » et d' « information ».

${ }^{9}$ Désormais $D H L F$.

${ }^{10}$ Du latin surgere au sens de « venir de bas en haut, s'élever » évoquant une idée de source.

${ }^{11}$ Avoir aucune part évoque essentiellement, comme nous le verrons, la notion de « participation ».

${ }^{12}$ Voir littérature sur les verbes supports.

13 Parmi les différentes définitions de la notion de grammaticalisation, Bertrand (2007: 41-42) a retenu celles données par De Mulder (2001 : 8), Marchello-Nizia (1999 : 159), le Dictionnaire de linguistique (1994, nouvelle édition $1999: 226)$, Traugott (1994: 1481) et Lehmann (1995: 9).
} 
${ }^{14}$ Une étude détaillée montrerait peut-être que nulle part «ailleurs, dans un autre endroit» (TLFi) et autre part « ailleurs », seraient des cas de grammaticalisation comme quelque part.

${ }^{15} \mathrm{http}: / /$ mauricianismes.wordpress.com/2011/07/01/aucune-part/

${ }_{16}$ Les exemples (77) et (78) ne sont pas d'exemples attestés mais plutôt des transformations en FM, état de langue dont nous avons la compétence, contrairement au français des siècles passés. 